Results Among 548 patients who were currently follow-up, 171 patients had $1^{\text {st }}$ LN episode. Of these, 87 patients (96.6\% female with mean age $29.5 \pm 10.8$ years) met the inclusion criteria. During $6.1 \pm 3.4$ years of observation, the incident of LN flare was $48.3 \%$. The mean time from CR to flare was 3.14 years $(\min 0.5$, $\max 9.5$ ). Logistic regression analysis revealed remaining dose of prednisololne $\geq 7.5 \mathrm{mg} /$ day after remission reduced incidence of renal flare (Odd ratio 0.26 (95\%CI 0.08-0.85), p=0.025), while demographic characteristics, clinical variables, and other treatments variables were not associated with incidence of LN flare.

Conclusions Although achieving CR with standard treatment, almost half of patients had LN flare within a few years. This study emphasise that maintenance phase in $\mathrm{LN}$ is crucial.

\section{USING DECISION TREE TO IDENTIFY THE ITP WITH HIGH PROBABILITY OF SLE DEVELOPMENT FROM A NATIONWIDE COHORT STUDY}

${ }^{1,2} \mathrm{TH}$ Li ${ }^{*}{ }^{3}$ YS Chang, ${ }^{4} \mathrm{CY}$ Tsai. ${ }^{1}$ Taichung Venterans general hospital - Chiayi branch, Division of Allergy- Immunology- and Rheumatology-Department of Medicine, Chiayi City, Taiwan R.O.C; ${ }^{2}$ National Yang-Ming University, Institute of Clinical Medicine, Taipei, Taiwan R.O.C; ${ }^{3}$ Shuang-Ho Hospital- Taipei Medical University, Division of Allergy- Immunologyand Rheumatology- Department of Internal Medicine, New Taipei City, Taiwan R.O.C; ${ }^{4}$ Taipei Veterans General Hospital, Division of Allergy- Immunology and RheumatologyDepartment of Internal Medicine, Taipei, Taiwan R.O.C

\subsection{6/lupus-2017-000215.407}

Background and aims Idiopathic thrombocytopenic purpura (ITP) is an immune-related thrombocytopenia which may herald the development of systemic lupus erythematosus (SLE), and thus regular following up has been suggested. Whereas widespread surveillance on all ITP patients would be time and cost-consuming; therefore identifying those with high probability of development of SLE among ITP patients should be more practical.

Methods We enrolled ITP patients without previous SLE diagnosis from the Taiwan National Health Insurance research database between 1997 and 2012 and identified those with SLE diagnosis during follow up. We also analysed the symptoms and comorbidities as well as the dose of average oral steroid to derive the decision trees, which classified the ITP patients with different probability of development of SLE.

Results A total of 10265 ITP patients were enrolled, among whom 80 patients developed SLE while following-up. The whole ITP patients were allocated to development group (7186 patients including 57 with SLE) and validation group (3079 patients including 23 with SLE); the former was used for derivation of the decision-tree based model (Figure 1) and the latter for validation of the previously mentioned model (Figure 2), and provided high sensitivity (78.2\%), specificity $(99.2 \%)$ and negative prediction value $(99.8 \%)$. To reduce the complexity, we also proposed another models with different complexity parameters (Figure 3).

Conclusions We derived different decision tree models exempt from the necessity of laboratory data and adequate for various clinical scenarios of ITP patients, among whom those with high probability of development of SLE could be identified.

\section{8 ANALYSIS OF INFLUENCING FACTORS ON QUALITY OF LIFE OF PATIENTS WITH SLE}

F Zeng, Q Xu, D Liu, G Deng, H Luo, X Zuo, Y Li*. Xiangya hospital - Central South University, Department of Rheumatology and Immunology, Changsha, China

\subsection{6/lupus-2017-000215.408}

Background and aims To investigate the influencing factors of quality of life (QOL) of patients with systemic lupus erythematosus (SLE).

Methods QOL of 104 SLE patients were investigated by SF36 scale (Chinese version). Depression, anxiety, social support, sleep quality and fatigue were evaluated by PHQ-9, GAD-7, social support rating scale (SSRS), Pittsburgh sleep quality index (PSQI) and VAS respectively. The demographic and clinical data were also recorded. SLE disease activity was assessed by SLEDAI.

Results In SF-36 scale, scores of SLE patients were lower than normal people in global score and in all dimensionalities $(\mathrm{p}<0.05)$. SLEDAI, PHQ-9, GAD-7, PQSI and fatigue correlated negatively with SF-36 scores $(\mathrm{p}<0.01)$. SSRS correlated positively with SF-36 scores $(\mathrm{p}<0.01)$. In binary logistic regression analyses, disease activity, anxiety, social support, sleep quality and fatigue were the independent determinants of QOL in SLE $\left(R^{2}=0.860, \mathrm{p}<0.01\right)$.

Conclusions QOL of SLE patients are lower than normal people. Disease activity, anxiety, social support, sleep quality and fatigue are the major influencing factors of QOL in SLE.

\section{DISEASE SEVERITY AND BURDEN IN JAPANESE PATIENTS WITH SYSTEMIC LUPUS ERYTHEMATOSUS FROM CLAIMS DATABASE}

${ }^{1} \mathrm{Y}$ Tanaka*, ${ }^{2} \mathrm{~T}$ Matsuki, ${ }^{2} \mathrm{~A}$ Mizukami, ${ }^{3} \mathrm{~A}$ Kobayashi, ${ }^{4} \mathrm{C}$ Ito. ${ }^{1}$ University of Occupational and Environmental Health, First Department of Internal Medicine- School of Medicine, Fukuoka, Japan; ${ }^{2}$ GlaxoSmithKline K.K, Vaccines Healthoutcomes Department, Tokyo, Japan; ${ }^{3}$ GlaxoSmithKline K.K, Biomedical Data Sciences Department, Tokyo, Japan; ${ }^{4}$ Japan Medical Data Centre Co. Ltd., Data Solution Division, Tokyo, Japan

\subsection{6/lupus-2017-000215.409}

Background and aims Disease burdens in Japanese patients with systemic lupus erythematosus (SLE) remain unclear. This study assessed disease burden of Japanese SLE patients with different disease activity in claims database.

\begin{tabular}{|c|c|c|}
\hline Total number & & 295 \\
\hline \multicolumn{3}{|l|}{ Disease severity } \\
\hline Mild & $N(\%)$ & $28(9.5)$ \\
\hline Moderate & $N(\%)$ & $134(45.4)$ \\
\hline Severe & $N(\%)$ & $133(45.1)$ \\
\hline Fomale & $N(\%)$ & $256(86.8)$ \\
\hline Age at the index & \pm SD) & $41.6 \pm 10.3$ \\
\hline \multicolumn{2}{|c|}{ Treatmont period at the index (mean year $=S D$ ) } & $6.5 \pm 6.0$ \\
\hline Principle insurer (full time worker) & $N(\%)$ & $107(36.3)$ \\
\hline Hospitalization during the study period & $N(\%)$ & $116(39.3)$ \\
\hline CNS lupus & $N(\%)$ & $12(4.5)$ \\
\hline Lupus nephritis & $N(\%)$ & $110(41.2)$ \\
\hline
\end{tabular}

